\title{
INVOLUCRAMIENTO ACADÉMICO: UNA ESCALA
}

\author{
Academic engagement: a scale
}

http://dx.doi.org/10.22235/pe.v10i1.1361

GUSTAVO PEÑA $T^{1}$

YOLANDA CAÑOTO $R^{2}$

LUISA ANGELUCCI B ${ }^{3}$

Universidad Católica Andrés Bello. Venezuela

Recibido: 16-09-2016

Correspondencia: Gustavo Peña T. Universidad Católica

Revisado: 14-10-2016

Andrés Bello. Venezuela. Correo electrónico: gpena@ucab.edu.ve

Aceptado: 03-11-2016

Resumen: El involucramiento académico (IA) se refiere a los recursos físicos y psicológicos que se dedican a la experiencia académica. Es predictor del aprendizaje y del desarrollo personal, y tiene tres componentes: conductual, cognitivo y afectivo. Para este trabajo se desarrolló una escala para medir la dimensión conductual del IA, que se administró a 906 estudiantes en Caracas, luego de un estudio piloto. Se realizó un análisis de confiabilidad, comportamiento de los ítems, estructura factorial y validez de criterio. Fue identificado un factor definido como personalidad resistente (PR) en el plano académico, que refleja el compromiso, control y asertividad del estudiante. Se concluye que la Escala de Involucramiento Académico y el factor PR son confiables, válidos y relevantes para la predicción de rendimiento académico.

Palabras clave: involucramiento académico, personalidad resistente, análisis psicométrico, rendimiento académico

\footnotetext{
${ }^{1}$ Vicerrector académico de la Universidad Católica Andrés Bello (UCAB), Caracas, Venezuela, para el período 2015-2019. Doctor en Psicología, investigador y docente en áreas de psicología experimental, metodología y estadística aplicada en las universidades UCAB, Central de Venezuela, Simón Bolívar, Lisandro Alvarado y Metropolitana. Ha sido director del Centro de Investigación y Evaluación Institucional (2007-2015), director del Doctorado en Psicología (2005- 2014), director de la Escuela de Psicología de la UCAB (1999-2005), director de los Estudios de Postgrado (1992-1993). Es autor de diversas publicaciones en el área de la psicología y en el campo de la estadística.

2 Magíster en Psicología. Investigadora del Centro de Investigación y Evaluación Institucional y profesora de la Escuela de Psicología de la UCAB, Caracas, Venezuela. Realiza proyectos de investigación y ha publicado libros, artículos y comunicaciones en congresos en las áreas de psicología general, rendimiento académico y psicología de la salud.

${ }^{3}$ Doctora en Psicología. Investigadora del Centro de Investigación y Evaluación Institucional y profesora de la Escuela de Psicología de la UCAB, Caracas, Venezuela. Profesora del Departamento de Ciencia y Tecnología del Comportamiento de la Universidad Simón Bolívar, Caracas, Venezuela. Realiza proyectos de investigación y ha publicado artículos y comunicaciones en congresos en las áreas de psicología social, psicometría y psicología de la salud.
} 


\begin{abstract}
Academic Engagement (AE) refers to the physical and psychological resources devoted to academic experience. It is a predictor of learning and personal development, and has three components: behavioral, cognitive and affective. A scale was developed to measure the behavioral dimension of $A E$. The scale was administered to 906 students in Caracas, after a pilot study, and analyses of reliability, item performance, factorial structure and criterion validity were performed. $A$ factor, defined as Personality Resistant (PR) at an academic level factor, was identified as a reflection of the commitment, control and assertiveness of the student. It is concluded that the scale factor $A E$ and $P R$ are reliable, valid and relevant for predicting academic performance.
\end{abstract}

Keywords: academic engagement, academic hardiness, psychometric analysis, academic performance

\title{
INTRODUCCIÓN
}

La educación es tanto un producto como un proceso. Cuando se evalúan los programas educativos y las instituciones, se suele enfatizar en el producto. Para estas evaluaciones se cuenta con diversos indicadores, que son ya tradicionales, como las calificaciones, número de estudiantes que obtienen diplomas, número de estudiantes que deben repetir o que se retiran (Pace, 1980). Estos indicadores suelen englobarse en el concepto de rendimiento académico.

El rendimiento académico es un componente clave para determinar si una institución ha alcanzado sus objetivos educativos. De allí lo esencial de los programas de evaluación que documentan este rendimiento (Commission on Higher Education, 1996; Rodríguez, Fita y Torrado, 2004). El proceso que conduce a este producto también tiene numerosas variables a considerar: rendimiento en el bachillerato, apoyo familiar y su nivel educativo, esfuerzo que realiza el alumno, estrategias docentes, programas de estudio, facilidades con las que cuenta la institución (Tejedor, 2003).

Entre los factores importantes al momento de explicar el rendimiento académico surge un conjunto de elementos que tienen que ver con el esfuerzo que realiza el estudiante para su logro: el número de horas que dedica al estudio, la asistencia a clase, la entrega de tareas. Todas estas conductas suelen englobarse en el concepto de involucramiento académico (IA). Este constructo aparece en la bibliografía desde hace más de 70 años, pero ha presentado cambios en su definición a lo largo del tiempo. Según Pascarella y Terenzini (1991), el IA ha recibido distintos nombres a lo largo de esta historia, entre ellos: tiempo en la tarea -término utilizado durante los años treinta del siglo XX-, calidad del esfuerzo (Pace, 1980), integración social y académica (Tinio, 2009), buenas prácticas (Chickering y Gamson, 1987), hasta el actual involucramiento (Kuh, 2009). 
En resumen, involucramiento académico se usa para referir a la cantidad de energía física y psicológica que el estudiante dedica a su experiencia académica. Un estudiante altamente involucrado es aquel que dedica una considerable cantidad de energía a estudiar, pasa mucho tiempo en el campus, participa activamente en las organizaciones estudiantiles e interactúa con frecuencia con otros estudiantes y con profesores (Astin, 1999).

Las características del IA, según la teoría del involucramiento de Astin (1999), son las siguientes:

- Se refiere a invertir energía en variados objetos físicos o psicológicos. Estos pueden ser generales (la experiencia del estudiante) o muy específicos (preparar un examen particular).

- Ocurre en un continuo; diferentes estudiantes manifiestan diferentes grados de involucramiento en el mismo objeto, y el mismo estudiante manifiesta diferentes grados en diferentes objetos.

- Tiene elementos cuantitativos (como la cantidad de horas que pasa estudiando) y cualitativos (compresión de las instrucciones).

- El desarrollo personal y el aprendizaje en cualquier programa educativo es directamente proporcional a la cantidad y calidad de involucramiento en ese programa.

- La efectividad de las políticas y prácticas educativas está directamente relacionada con su capacidad de incrementar el involucramiento de los estudiantes.

Otra definición de involucramiento lo asume como un estado mental positivo, relacionado con el trabajo y caracterizado por tres elementos: vigor (altos niveles de energía y resistencia mental), dedicación (alta implicación laboral) y absorción (alto estado de concentración e inmersión). Este estado afectivo-cognitivo es persistente y no está focalizado en un objeto o situación particular. El vigor y la absorción se relacionan positivamente con el rendimiento académico, determinado a través del número de exámenes aprobados (Parra, 2010).

En las diferentes investigaciones, el involucramiento aparece conformado por dos o tres componentes. Los modelos que incluyen dos elementos, los definen como uno conductual y otro emocional o afectivo. Los de tres componentes incluyen un elemento cognitivo (Appleton, Christenson y Furlong, 2008). Para Kuh, Kinzie, Schuh y Whitt (2005), así como para Whitt, Asel y Edvalson (2008), el involucramiento está compuesto por dos elementos: a) la cantidad de tiempo y 
esfuerzo que los estudiantes ponen en sus estudios, además de otras actividades que llevan a las experiencias y resultados de éxito; b) las formas en que una institución asigna sus recursos - humanos y de otro tipo- y organiza las oportunidades de aprendizaje y servicios para animar a los estudiantes a participar y beneficiarse de ellas. Trowler y Trowler (2011), por su parte, plantean que el IA tiene tres dimensiones: conductual, cognitiva y afectiva. En cada caso, a su vez, tienen manifestaciones congruentes (positivas) u opuestas (negativas).

A partir de estas definiciones, se plantea que el involucramiento es la inversión de tiempo, esfuerzo y otros recursos relevantes, por parte del estudiante y de la institución, en el intento de optimizar la experiencia y mejorar el resultado del aprendizaje, el desarrollo del estudiante y la reputación de la institución. Como puede verse, en esta definición se involucra a la institución, no solo al estudiante. En el presente artículo se enfatiza la primera de las dimensiones antes señaladas.

Relacionada con el concepto de IA, aparece la variable de la personalidad resistente $(\mathrm{PR})$, que se entiende como "una constelación de características de personalidad que funcionan como un recurso de resistencia contra los eventos estresantes de la vida diaria" (Kobasa, 1979, p. 414). Este constructo consta de tres componentes: compromiso, control y asertividad (Kobasa, Maddi y Kahn, 1982). En el individuo resistente, el compromiso se expresa como una tendencia a involucrarse (en contraposición a la alienación) en las actividades de la vida como el trabajo, la familia, el yo o los pasatiempos (Nowack, 1991). Las personas comprometidas tienen un sentido generalizado de propósito que les permite identificarse y encontrar eventos significativos en cosas y personas de su entorno.

El control se expresa como una tendencia a sentir y actuar como si se tuviera influencia (en lugar de sentirse impotente) de cara a las contingencias variadas de la vida (Averill, 1973; Phares, 1976), lo que da al individuo una sensación de autonomía y de poder para afectar el propio futuro. Este concepto implica una percepción de sí mismo como alguien que puede tener influencia a través del ejercicio de la imaginación, el conocimiento, la habilidad y la elección. El tercer componente de resistencia, desafío, se expresa como la creencia de que el cambio, más que la estabilidad, es lo normal en la vida y la anticipación del cambio es un estímulo para el crecimiento y no una amenaza a la seguridad. Los desafíos se entienden como estímulos, específicamente porque son cambios que requieren reajuste (Kobasa et al., 1982 y Judkins, 2001).

Esta estrecha relación entre IA y PR hace pensar que ambos aspectos tienen importante influencia sobre el rendimiento académico, como apuntan Dehyadegary, Yaacob, Juhari y Talib (2011). Estos autores reportan correlaciones bivariadas que reflejan una asociación fuerte y significativa entre el rendimiento académico e IA $(r=0,69, p<0,05)$. Sus resultados coinciden con estudios previos - por ejemplo, Sirin 
y Sirin (2004), Sbrocco (2009), Wang y Holcombe (2010) - que encuentran correlaciones entre IA y éxito académico. Según esta perspectiva, el tiempo y la energía que los estudiantes dedican a actividades relacionadas con sus propósitos educativos son el mejor predictor de su aprendizaje y desarrollo personal (Astin, 1993; Pace, 1980; Pascarella y Terenzini, 1991). Casuso-Holgado et al. (2013), en una muestra de 911 estudiantes de la Universidad de Málaga (España), también reportan que el IA tiene influencia positiva sobre el rendimiento académico, además de encontrar relaciones con otras variables como satisfacción, autoeficacia y estilos de aprendizaje.

Adicionalmente, se ha establecido que ciertas prácticas institucionales también pueden conducir a altos niveles de participación de los estudiantes -Astin, 1991; Chickering y Reisser, 1993; Kuh, Schuh, Whitt y Associates, 1991; Pascarella y Terenzini, 1991 - y actuar como posible antídoto de la deserción. El abandono de los estudios universitarios es un fenómeno que puede alcanzar cifras tan alarmantes como las que reportan Abello et al. (2012), quienes estiman que un $50 \%$ de los estudiantes chilenos que ingresan a la universidad no logra completar sus estudios. El IA suele relacionarse con el abandono de los estudios o la deserción, en el sentido de que los estudiantes con mayor involucramiento son los que logran graduarse (Appleton, Christenson y Furlong, 2008). Esta afirmación se basa en que la deserción no es un fenómeno repentino, sino que es un proceso gradual de desconexión con la institución y los estudios en general. Aumentar el IA interviene en las primeras etapas de esta desconexión y enfoca la atención en variables alternativas que ayudan a completar los grados académicos.

Por otro lado, el IA está relacionado también con el nivel de satisfacción que muestran los estudiantes con sus actividades. Parada y Pérez (2014) encontraron, en su investigación realizada entre estudiantes de odontología en universidades chilenas, que el mayor IA lo muestran las estudiantes de sexo femenino, con mejor rendimiento en las asignaturas relacionadas con las matemáticas de los años superiores y que presentaban también mayor satisfacción con la universidad y sus estudios en general.

Se han construido numerosos instrumentos para la medida del IA, como el College Student Experiences Questionnaire (CSEQ; Pace, 1980), que mide la calidad del esfuerzo o el National Survey of Student Engagement (NSSE; Kuh, 2009), un instrumento de aplicación nacional en los Estados Unidos que cuenta con larga y amplia investigación. Esta escala recoge información en cinco áreas: a) aprendizaje activo-colaborativo, b) contacto estudiante-facultad, c) nivel de reto académico, d) experiencias educativas enriquecedoras y e) apoyo ambiental del campus (Kuh, 2009). Se dispone también de escalas desarrolladas en español, como el cuestionario de implicación académica de Abello et al. (2012), quienes 
también encontraron cinco dimensiones: a) apoyo personal obtenido, b) actividad de la vocación, c) comportamiento social, d) recursos ofrecidos por la institución y e) aprendizaje activo.

El objetivo del presente estudio es construir un instrumento para la medida del IA que se adapte a las características de la población universitaria de la Universidad Católica Andrés Bello (UCAB) en Caracas, Venezuela. Concretamente, para el desarrollo de esta escala, se partió del repertorio de 102 ítems propuestos por Tinio (2009) para evaluar compromiso académico. Así, los objetivos específicos de este trabajo consistieron en realizar análisis de la confiabilidad, del comportamiento de los ítems, estructura factorial y validez de criterio de la Escala de Involucramiento Académico desarrollada.

\section{MÉTODO}

Se utilizó como punto de partida la Escala de Compromiso Académico de Tinio (2009), compuesta por 102 ítems. A partir de un estudio piloto, se seleccionaron aquellos 50 que, según el criterio de jueces expertos, resultaron más idóneos a los fines de evaluar IA; adicionalmente, se evitó el solapamiento de sus contenidos. Además, se solicitó a los participantes que indicaran la carrera que cursaban, el año o semestre, el sexo y el promedio de calificaciones obtenido en la UCAB hasta ese momento.

\section{Muestra}

La escala se aplicó a una muestra de estudiantes universitarios de la UCAB, de $2^{\circ}$ y $4^{\circ}$ año, y los semestres análogos, entre marzo y abril del 2012. También se incluyó una pequeña muestra de alumnos de la Universidad Metropolitana (UNIMET, Caracas). El total de 906 cuestionarios se distribuyeron, de acuerdo a la carrera y al sexo, como se indica en la tabla $n . \stackrel{\circ}{1}$. Fueron eliminados 22 cuestionarios porque no indicaban el sexo del alumno. 
Tabla N.o 1

Distribución de los estudiantes por sexo, carrera y universidad

\begin{tabular}{ccccc}
\hline Escuela & Hombres & Mujeres & Total & Universidad \\
\hline Administración y contaduría & 54 & 57 & 111 & \\
Comunicación social & 17 & 132 & 149 & \\
Derecho & 26 & 66 & 92 & UCAB \\
Ingeniería & 290 & 135 & 425 & \\
Psicología & 14 & 93 & 107 & UNIMET \\
Total & 401 & 483 & 884 & \\
& & & & \\
\hline
\end{tabular}

Fuente: Elaboración propia de los autores (2016)

\section{RESULTADOS}

Los datos fueron tabulados y procesados con el programa SPSS 18.0. Para el análisis de la consistencia interna, se calculó el coeficiente de confiabilidad alfa de Cronbach, que arrojó un valor de 0,896 y el coeficiente theta de 0,91, lo que muestra una alta consistencia interna entre los ítems. Las correlaciones de los ítems con el puntaje total fueron positivas y superiores a 0,30, a excepción de los ítems 2, 4, 6, $10,17,19,20,35$ y 42 (superiores a 0,25 ) y el 49 (cercano a 0,23 ), lo que es aceptable para este tipo de cuestionario, según los criterios que se utilizan en estas investigaciones.

Para determinar la estructura factorial de la escala, se procedió a realizar un análisis de componentes principales con rotación varimax de todos los reactivos (ACP1). Se seleccionaron los factores con autovalores superiores a 1,5, con lo cual se obtuvo una solución factorial integrada por seis componentes que, en conjunto, explican el 39,8\% de la varianza total. Para seleccionar los ítems que componían cada factor, se fijó como criterio una carga factorial por encima de 0,35.

Por el significado de los ítems agrupados, el primer factor se denominó apego a la universidad (18,16\% de la varianza explicada); el segundo, atención en clase (6,14\%); el tercero, participación activa (5,07\%); el cuarto, dedicación (4,03\%); el quinto, focalización en la tarea (3,40 \%) y el sexto, integración social (3\%). 
Tabla N.O2

Estructura factorial de la Escala de IA (recorrido de respuesta de los ítems: 1 a 4)

\begin{tabular}{|c|c|c|c|c|c|c|c|c|}
\hline Componente & $\mathrm{N}^{\circ}$ & & $h_{A C P 1}$ & $x$ & $S$ & $r_{\text {it }}$ & $\alpha_{-i}$ & hACP2 \\
\hline \multirow{10}{*}{ 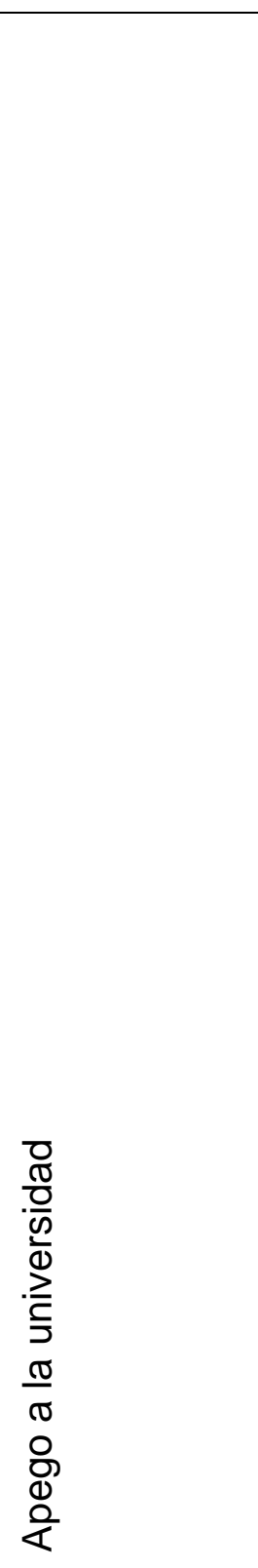 } & 47 & $\begin{array}{l}\text { Estoy satisfecho con la } \\
\text { calidad de la educación en } \\
\text { mi universidad }\end{array}$ & 0,724 & 3,32 & 0,750 & 0,372 & 0,894 & \\
\hline & 34 & $\begin{array}{l}\text { Siento que pertenezco a la } \\
\text { universidad }\end{array}$ & 0,674 & 3,46 & 0,751 & 0,412 & 0,894 & \\
\hline & 1 & $\begin{array}{l}\text { Estoy orgulloso de ser un } \\
\text { estudiante de esta } \\
\text { universidad }\end{array}$ & 0,666 & 3,64 & 0,665 & 0,339 & 0,895 & \\
\hline & 5 & $\begin{array}{l}\text { En la universidad hay un } \\
\text { buen ambiente de } \\
\text { aprendizaje }\end{array}$ & 0,602 & 3,29 & 0,711 & 0,321 & 0,895 & \\
\hline & 12 & $\begin{array}{l}\text { Me agrada venir a la } \\
\text { universidad }\end{array}$ & 0,596 & 3,24 & 0,745 & 0,444 & 0,893 & $0,684^{2}$ \\
\hline & 31 & $\begin{array}{l}\text { Aprendo mucho en la } \\
\text { universidad }\end{array}$ & 0,598 & 3,51 & 0,615 & 0,470 & 0,893 & \\
\hline & 50 & $\begin{array}{l}\text { Quiero que otras personas } \\
\text { vengan a estudiar en mi } \\
\text { universidad }\end{array}$ & 0,542 & 3,38 & 0,863 & 0,366 & 0,894 & \\
\hline & 17 & $\begin{array}{l}\text { Me siento seguro en la } \\
\text { universidad }\end{array}$ & 0,539 & 3,40 & 0,756 & 0,254 & 0,896 & $0,66^{2}$ \\
\hline & 33 & $\begin{array}{l}\text { Aprecio el trabajo arduo } \\
\text { que realizan los profesores }\end{array}$ & 0,451 & 3,25 & ,732 & 0,442 & 0,893 & $0,59^{2}$ \\
\hline & 46 & $\begin{array}{l}\text { Creo que los profesores } \\
\text { son considerados }\end{array}$ & 0,388 & 2,55 & 0,813 & 0,385 & 0,894 & \\
\hline \multirow{8}{*}{ 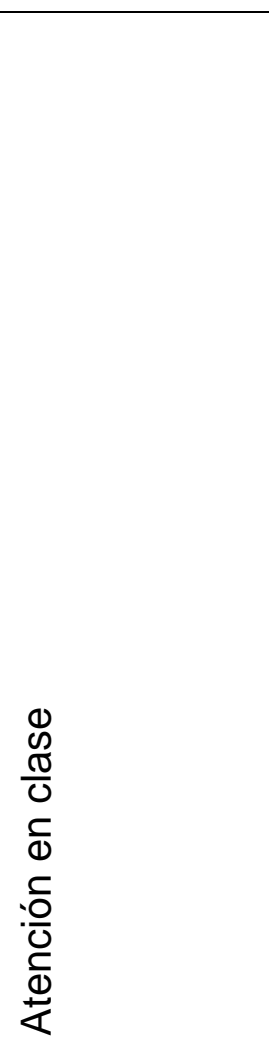 } & 27 & $\begin{array}{l}\text { Escucho atentamente las } \\
\text { clases }\end{array}$ & 0,689 & 3,32 & 0,637 & 0,449 & 0,894 & \\
\hline & 32 & $\begin{array}{l}\text { Me concentro en lo que el } \\
\text { profesor está explicando en } \\
\text { clase }\end{array}$ & 0,683 & 3,29 & 0,652 & 0,483 & 0,893 & \\
\hline & 3 & $\begin{array}{l}\text { Estoy atento durante las } \\
\text { discusiones en clase }\end{array}$ & 0,598 & 3,23 & 0,648 & 0,451 & 0,894 & \\
\hline & 22 & Evito distraerme en clase & 0,579 & 2,83 & 0,792 & 0,484 & 0,893 & \\
\hline & 23 & $\begin{array}{l}\text { Sigo las reglas del salón de } \\
\text { clases }\end{array}$ & 0,534 & 3,47 & 0,669 & 0,375 & 0,894 & \\
\hline & 8 & $\begin{array}{l}\text { Tengo buenas relaciones } \\
\text { con los profesores }\end{array}$ & 0,409 & 3,45 & 0,611 & 0,309 & 0,895 & \\
\hline & 4 & $\begin{array}{l}\text { Me mantengo concentrado } \\
\text { cuando respondo un examen }\end{array}$ & 0,395 & 3,48 & 0,628 & 0,289 & 0,895 & $0,37^{2}$ \\
\hline & 7 & Asisto a las clases & 0,368 & 3,77 & 0,480 & 0,311 & 0,895 & \\
\hline
\end{tabular}




\begin{tabular}{|c|c|c|c|c|c|c|c|c|}
\hline Componente & $\mathrm{N}^{\circ}$ & & hACP1 & $\mathrm{X}$ & $S$ & $r_{\text {it }}$ & $\alpha_{-i}$ & $\mathrm{~h}_{\mathrm{ACP} 2}$ \\
\hline & 41 & $\begin{array}{l}\text { Doy mi punto de vista } \\
\text { acerca del tema en las } \\
\text { discusiones en clase }\end{array}$ & 0,706 & 2,59 & 0,919 & 0,469 & 0,893 & $.735^{1}$ \\
\hline & 26 & $\begin{array}{l}\text { Hago preguntas al profesor } \\
\text { cuando no entiendo el } \\
\text { contenido de la clase }\end{array}$ & 0,574 & 2,95 & 0,865 & 0,373 & 0,894 & \\
\hline & 36 & $\begin{array}{l}\text { Me involucro en actividades } \\
\text { extracurriculares }\end{array}$ & 0,557 & 1,89 & 1,002 & 0,333 & 0,895 & $0,630^{1}$ \\
\hline & 20 & $\begin{array}{l}\text { Corrijo al profesor si me } \\
\text { parece que hay algo mal en } \\
\text { la clase }\end{array}$ & 0,556 & 2,28 & 0,986 & 0,219 & 0,897 & \\
\hline & 21 & $\begin{array}{l}\text { Soy un miembro activo de } \\
\text { mi escuela }\end{array}$ & 0,542 & 1,74 & 0,936 & 0,376 & 0,894 & \\
\hline & 29 & $\begin{array}{l}\text { Trato de responder a las } \\
\text { preguntas del profesor }\end{array}$ & 0,533 & 2,87 & 0,820 & 0,469 & 0,893 & $0,72^{1}$ \\
\hline & 13 & $\begin{array}{l}\text { Cuanto más difícil sea la } \\
\text { tarea, mejor para mí }\end{array}$ & 0,456 & 2,20 & 0,864 & 0,352 & 0,894 & \\
\hline & 48 & $\begin{array}{l}\text { Me empeño en ir más allá } \\
\text { de lo que se espera de mí } \\
\text { en la universidad }\end{array}$ & 0,418 & 2,98 & 0,777 & 0,555 & 0,892 & \\
\hline 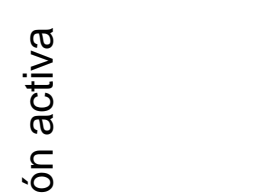 & 11 & $\begin{array}{l}\text { Me gusta cuando me } \\
\text { asignan tareas para hacer } \\
\text { en casa }\end{array}$ & 0,387 & 2,17 & 0,910 & 0,396 & 0,894 & \\
\hline 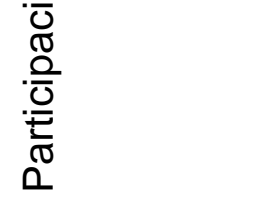 & 6 & $\begin{array}{l}\text { Contacto al profesor } \\
\text { cuando tengo que aclarar } \\
\text { algo de la materia }\end{array}$ & 0,356 & 2,63 & 0,923 & 0,292 & 0,895 & \\
\hline \multirow{7}{*}{ 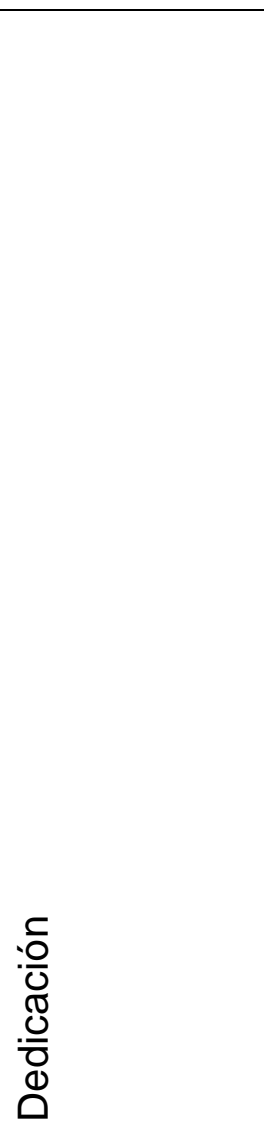 } & 15 & $\begin{array}{l}\text { Estudio con antelación para } \\
\text { las evaluaciones o } \\
\text { exámenes }\end{array}$ & 0,669 & 3,12 & 0,745 & 0,398 & 0,894 & \\
\hline & 44 & $\begin{array}{l}\text { Dedico horas extras al } \\
\text { estudio }\end{array}$ & 0,6 & 2,77 & 0,871 & 0,433 & 0,893 & \\
\hline & 30 & $\begin{array}{l}\text { Me preparo para las } \\
\text { pruebas, exámenes o } \\
\text { evaluaciones }\end{array}$ & 0,599 & 3,55 & 0,623 & 0,381 & 0,894 & \\
\hline & 9 & $\begin{array}{l}\text { Me esfuerzo por ser un } \\
\text { excelente estudiante }\end{array}$ & 0,557 & 3,26 & 0,697 & 0,493 & 0,893 & \multirow[t]{4}{*}{$0,49^{2}$} \\
\hline & 18 & $\begin{array}{l}\text { Hago mi mayor esfuerzo en } \\
\text { todas las actividades } \\
\text { académicas }\end{array}$ & 0,521 & 3,27 & 0,697 & 0,502 & 0,893 & \\
\hline & 25 & $\begin{array}{l}\text { Las tareas para la casa las } \\
\text { hago lo antes posible }\end{array}$ & 0,436 & 2,66 & 0,877 & 0,434 & 0,893 & \\
\hline & 24 & $\begin{array}{l}\text { Presento a tiempo los } \\
\text { trabajos y las asignaciones }\end{array}$ & 0,389 & 3,73 & 0,500 & 0,318 & 0,895 & \\
\hline
\end{tabular}




\begin{tabular}{|c|c|c|c|c|c|c|c|c|}
\hline \multirow[t]{2}{*}{ Componente } & \multicolumn{2}{|l|}{$\mathrm{N}^{\circ}$} & \multirow{2}{*}{$\begin{array}{l}\text { hACP1 } \\
0,368\end{array}$} & \multirow{2}{*}{$\begin{array}{l}x \\
3,36\end{array}$} & \multirow{2}{*}{$\begin{array}{l}S \\
0,748\end{array}$} & \multirow{2}{*}{$\begin{array}{l}\text { rit } \\
0,389\end{array}$} & \multirow{2}{*}{$\begin{array}{l}\alpha-i \\
0,894\end{array}$} & \multirow[t]{2}{*}{$\mathrm{h}_{\mathrm{ACP} 2}$} \\
\hline & 38 & $\begin{array}{l}\text { Mi prioridad principal es la } \\
\text { universidad }\end{array}$ & & & & & & \\
\hline \multirow{9}{*}{ 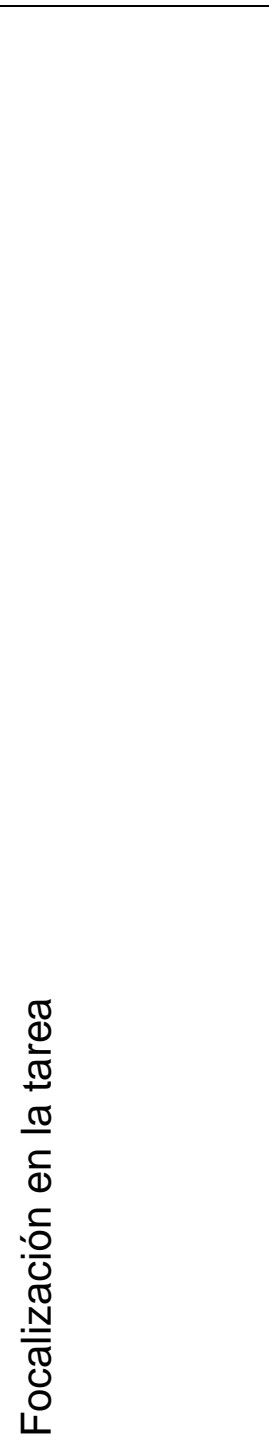 } & 43 & $\begin{array}{l}\text { No me salgo del salón } \\
\text { cuando no me gusta la } \\
\text { clase }\end{array}$ & 0,675 & 2,66 & 1,148 & 0,315 & 0,896 & \\
\hline & 42 & $\begin{array}{l}\text { No sueño despierto durante } \\
\text { las clases }\end{array}$ & 0,574 & 2,43 & 0,965 & 0,290 & 0,895 & \\
\hline & 45 & $\begin{array}{l}\text { No me aburro durante las } \\
\text { clases }\end{array}$ & 0,529 & 2,47 & 0,750 & 0,398 & 0,894 & \\
\hline & 16 & $\begin{array}{l}\text { No me doy por vencido } \\
\text { cuando la tarea asignada } \\
\text { es difícil }\end{array}$ & 0,511 & 2,88 & 0,939 & 0,397 & 0,894 & $0,61^{4}$ \\
\hline & 49 & $\begin{array}{l}\text { No entrego los trabajos si } \\
\text { están incompletos }\end{array}$ & 0,498 & 2,66 & 1,131 & 0,235 & 0,897 & $0,78^{4}$ \\
\hline & 40 & $\begin{array}{l}\text { No me distraigo con mis } \\
\text { compañeros durante la } \\
\text { clase }\end{array}$ & 0,497 & 2,61 & 0,825 & 0,382 & 0,894 & $0,55^{4}$ \\
\hline & 14 & $\begin{array}{l}\text { No renuncio fácilmente a } \\
\text { hacer las tareas }\end{array}$ & 0,475 & 2,90 & 0,898 & 0,365 & 0,894 & \\
\hline & 10 & $\begin{array}{l}\text { Creo que no debería } \\
\text { cambiarme a otra } \\
\text { universidad }\end{array}$ & 0,314 & 3,09 & 1,236 & 0,272 & 0,897 & \\
\hline & 35 & $\begin{array}{l}\text { Me incomoda fallar algunas } \\
\text { veces en la universidad }\end{array}$ & 0,302 & 3,30 & 0,831 & 0,290 & 0,895 & \\
\hline \multirow{5}{*}{ 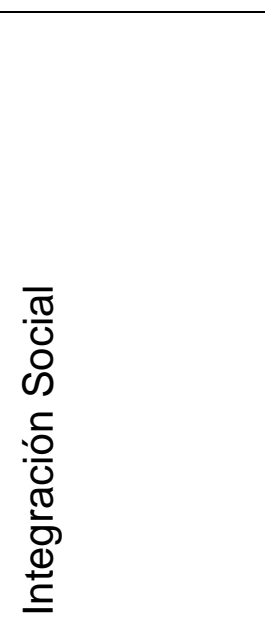 } & 2 & $\begin{array}{l}\text { Tengo buena relación con } \\
\text { mis compañeros }\end{array}$ & 0,579 & 3,54 & 0,618 & 0,273 & 0,895 & \\
\hline & 19 & Me gusta trabajar en grupo & 0,562 & 3,08 & 0,885 & 0,258 & 0,896 & $0,78^{3}$ \\
\hline & 28 & $\begin{array}{l}\text { Soy tomado en cuenta por } \\
\text { mis compañeros de clase }\end{array}$ & 0,552 & 3,17 & 0,736 & 0,407 & 0,894 & \\
\hline & 37 & $\begin{array}{l}\text { Participo en grupos de } \\
\text { estudio }\end{array}$ & 0,504 & 2,56 & 1,038 & 0,325 & 0,895 & $0,69^{3}$ \\
\hline & 39 & $\begin{array}{l}\text { Ayudo a mis compañeros } \\
\text { que no entienden las clases }\end{array}$ & 0,434 & 3,04 & 0,829 & 0,384 & 0,894 & \\
\hline
\end{tabular}

Fuente: Elaboración propia de los autores (2016) 
En la tabla $n .03$ se puede apreciar que, al emplear una escala del 0 al 20 , los participantes consideran que realizan de forma moderada-alta los comportamientos implicados en los factores apego a la universidad, atención en clase y dedicación (Media $=15,82,15,68$ y 15,09, respectivamente). Reportan una integración social media-baja (Media $=14,09$ ) con los compañeros dentro de la universidad, y más bien baja focalización en la tarea y baja participación activa en los estudios (Media $=12,6$ y 10,24 , respectivamente).

Tabla n. 03

Estadísticos generales de las puntuaciones en los componentes del IA

\begin{tabular}{lccccc}
\hline Componente & $\mathrm{N}$ & Mín. & Máx. & Media & $\mathrm{S}$ \\
\hline Apego a la Universidad & 887 & 6,00 & 20 & 15,82 & 2,72 \\
Atención en clase & 892 & 5,00 & 20 & 15,68 & 2,66 \\
Participación & 875 & 0,67 & 20 & 10,24 & 3,28 \\
Dedicación & 889 & 5,00 & 20 & 15,09 & 2,62 \\
Focalización en la tarea & 870 & 1,48 & 20 & 12,06 & 3,12 \\
Integración social & 894 & 1,33 & 20 & 14,09 & 3,29
\end{tabular}

Fuente: Elaboración propia de los autores (2016)

Se observa que, si bien no se agrupan según alguna dimensión o componente del IA, los ítems con mayor promedio se refieren en su mayoría a aspectos relacionados con la atención en clase y apego a la universidad; mientras que los ítems con menor promedio, y que por tanto identifican menos al estudiante, están en su mayoría referidos a las dimensiones de participación activa y focalización en la tarea.

Con la finalidad de evaluar en qué medida el rendimiento académico (RA) puede ser predicho por las dimensiones del IA, se calculó una regresión múltiple del RA con base en las dimensiones del IA, considerando el sexo de los estudiantes como otra variable predictora.

Como se puede observar en la tabla $n .04$, entre 460 estudiantes, el modelo explica significativamente el $15 \%$ de la varianza total de la variable predicha $\left(R=0,393, R^{2}=0,154, F=11,8, p=0,00\right)$. En particular, el sexo predice significativamente al rendimiento académico, donde las mujeres presentan mayor 
rendimiento que los hombres $(\beta=0,31, p=0,00)$. Ninguna dimensión del IA predice el rendimiento académico reportado por los estudiantes.

Tabla N.O4

Regresión del rendimiento académico a partir de los componentes del ACP con todos los ítems $(n=50)$ de la prueba

\begin{tabular}{|c|c|c|c|}
\hline & Beta & $t$ & Sig. \\
\hline (Intercepto) & & 17,083 & 0,000 \\
\hline Apego & $-0,077$ & $-1,354$ & 0,177 \\
\hline Atención & 0,076 & 1,258 & 0,209 \\
\hline Participación & 0,085 & 1,492 & 0,136 \\
\hline Dedicación & 0,108 & 1,608 & 0,108 \\
\hline Focalización & 0,101 & 1,870 & 0,062 \\
\hline Integración & $-0,103$ & $-1,783$ & 0,075 \\
\hline Sexo & 0,309 & 6,673 & $0,000^{*}$ \\
\hline
\end{tabular}

Fuente: Elaboración propia de los autores (2016) 
Con el fin de analizar con mayor detalle la predicción de rendimiento a partir de la Escala de IA, se realizó una regresión múltiple del rendimiento a partir de cada uno de los 50 ítems. A partir del análisis, se encontró que trece ítems del IA predicen el rendimiento académico $(4,9,12,16,17,19,29,33,36,37,40,41$ y 49). De ellos, como se puede ver en la tabla $n . \stackrel{0}{2}$, tres pertenecen a la dimensión de apego, uno de ellos a la dimensión de atención, tres a la participación, uno a la dedicación, tres a la focalización y dos a la integración. Con estos ítems, que son los que mejor predicen el rendimiento académico, se realizó un análisis de componentes principales (ACP2) y se obtuvieron cuatro dimensiones en las que los ítems sobre apego, atención y dedicación se unen en un solo factor (ahora factor 2), mientras que los otros se ubicaron donde originalmente estaban: participación (factor 1), integración (factor 3 ) y focalización (factor 4).

Posteriormente, se realizó un análisis de diferencia de medias para cada uno de los componentes del IA (ACP1) mediante un análisis de varianza factorial $2 \times 5$ (sexo $x$ escuela), con el objetivo de examinar las diferencias en estas dimensiones en función del sexo y la carrera (ver tabla n.․ 5). Se evidencia que el sexo es significativo en participación y en dedicación, en el sentido de que los hombres reportan una mayor participación activa (hombres $=10,37$ y mujeres $=10,10$ ) y las mujeres una mayor dedicación a los estudios (mujeres $=15,67$ y hombres $=14,46$ ).

Asimismo, se observa que la escuela resulta significativa para el apego a la universidad y la atención en clase, de manera que los estudiantes de comunicación social y derecho presentan un mayor apego ( 16,89 y 16,49 , respectivamente), y los estudiantes de ingeniería, psicología y administración reportan el menor apego $(15,27 ; 15,79$ y 16,06 , respectivamente). Por otra parte, los estudiantes de derecho, psicología y comunicación social manifiestan poseer la mayor atención en clase $(16,34 ; 16,03$ y 16,02 , respectivamente), mientras que los de ingeniería y administración la menor atención (15,34 y 15,77, respectivamente). El efecto de interacción sexo por escuela no resultó significativo (tabla $n$. ․ 5) 
Tabla N. O 5

ANOVA de las dimensiones del IA (ACP 1) en función del sexo y escuela $(2 \times 5)$ para todas las dimensiones

\begin{tabular}{|c|c|c|c|c|c|c|c|c|c|c|c|c|}
\hline & Focalización & & Apego & & Integración & & Atención & & Participación & & Dedicación & \\
\hline Fuente & $\mathrm{F}$ & $\mathrm{P}$ & $\mathrm{F}$ & $\mathrm{P}$ & $\mathrm{F}$ & $P$ & $\mathrm{~F}$ & $\mathrm{P}$ & $\mathrm{F}$ & $\mathrm{P}$ & $\mathrm{F}$ & $\mathrm{P}$ \\
\hline Sexo & 0,04 & 0,85 & 0,22 & 0,63 & 2,21 & 0,13 & 0,95 & 0,33 & 4,08 & $0,04^{*}$ & 25,1 & 0,00 * \\
\hline Escuela & 2,29 & 0,06 & 5,67 & $0,00^{*}$ & 0,68 & 0,61 & 2,57 & $0,04^{*}$ & 1,34 & 0,24 & 0,94 & 0,43 \\
\hline Sexo x escuela & 0,84 & 0,49 & 0,61 & 0,65 & 0,58 & 0,87 & 0,96 & 0,42 & 1,54 & 0,18 & 0,39 & 0,81 \\
\hline
\end{tabular}

Fuente: Elaboración propia de los autores (2016) 
En un análisis adicional, se ubicaron algunos ítems cuya redacción apunta a lo que se denomina personalidad resistente o academic hardiness, por lo cual se procedió a distinguir estos elementos en una subescala particular y estudiar su comportamiento a partir del análisis de confiabilidad y estructura factorial.

Como se puede ver en la tabla n.․ 6 , se obtuvieron las tres dimensiones de personalidad resistente referidas por Kobasa et al. (1982) a partir de un análisis de componentes principales, lo que apunta a la validez de este constructo. Asimismo, se observa una alta confiabilidad, con un alfa de Cronbach $=0,73$ y un theta $=0,75$.

Tabla N. 06

Estructura factorial de la subescala de hardiness académico

\begin{tabular}{|c|c|c|c|c|c|c|c|}
\hline Componente & $\mathrm{N}^{\circ}$ & Confiabilidad: $\alpha=.73$ / $\theta=.75$ & $\mathrm{~h}_{1}$ & $\mathrm{X}$ & S & $r_{\text {it }}$ & $\alpha-i$ \\
\hline \multirow[b]{4}{*}{ 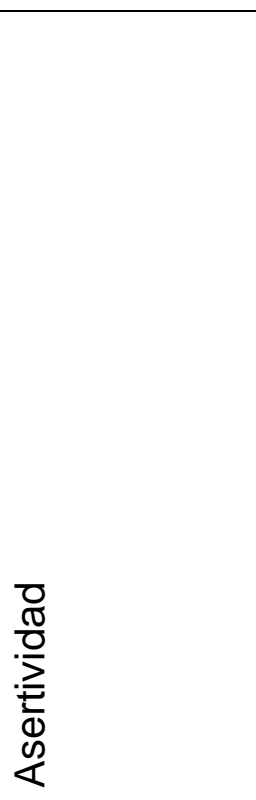 } & 26 & $\begin{array}{l}\text { Hago preguntas al profesor } \\
\text { cuando no entiendo el contenido } \\
\text { de la clase }\end{array}$ & 0,784 & 2,95 & 0,860 & 0,429 & 0,706 \\
\hline & 41 & $\begin{array}{l}\text { Doy mi punto de vista acerca del } \\
\text { tema en las discusiones en } \\
\text { clase }\end{array}$ & 0,718 & 2,59 & 0,925 & 0,484 & 0,698 \\
\hline & 20 & $\begin{array}{l}\text { Corrijo al profesor si me parece } \\
\text { que hay algo mal en la clase }\end{array}$ & 0,675 & 2,28 & 0,991 & 0,279 & 0,727 \\
\hline & 6 & $\begin{array}{l}\text { Contacto al profesor cuando } \\
\text { tengo que aclarar algo de la } \\
\text { materia }\end{array}$ & 0,507 & 2,63 & 0,930 & 0,300 & 0,723 \\
\hline \multirow[b]{4}{*}{$\begin{array}{l}\overline{0} \\
\text { 온 } \\
\text { Oे }\end{array}$} & 14 & $\begin{array}{l}\text { No renuncio fácilmente a hacer } \\
\text { las tareas }\end{array}$ & 0,800 & 2,91 & 0,899 & 0,403 & 0,709 \\
\hline & 16 & $\begin{array}{l}\text { No me doy por vencido cuando } \\
\text { la tarea asignada es difícil }\end{array}$ & 0,507 & 2,87 & 0,948 & 0,409 & 0,708 \\
\hline & 43 & $\begin{array}{l}\text { No me salgo del salón cuando } \\
\text { no me gusta la clase }\end{array}$ & 0,484 & 2,65 & 1,149 & 0,276 & 0,731 \\
\hline & 13 & $\begin{array}{l}\text { Cuanto más difícil sea la tarea, } \\
\text { mejor para mí }\end{array}$ & 0,465 & 2,20 & 0,861 & 0,361 & 0,715 \\
\hline \multirow[b]{4}{*}{ 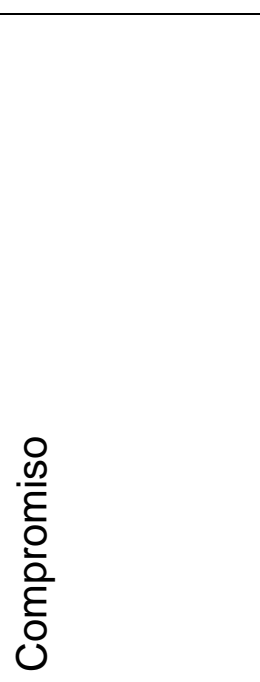 } & 38 & $\begin{array}{l}\text { Mi prioridad principal es la } \\
\text { universidad }\end{array}$ & 0,695 & 3,36 & 0,743 & 0,252 & 0,727 \\
\hline & 18 & $\begin{array}{l}\text { Hago mi mayor esfuerzo en } \\
\text { todas las actividades } \\
\text { académicas }\end{array}$ & 0,651 & 3,27 & 0,698 & 0,400 & 0,712 \\
\hline & 44 & Dedico horas extras al estudio & 0,622 & 2,78 & 0,868 & 0,383 & 0,712 \\
\hline & 48 & $\begin{array}{l}\text { Me empeño en ir más allá de lo } \\
\text { que se espera de mí en la } \\
\text { universidad }\end{array}$ & 0,621 & 2,99 & 0,777 & 0,475 & 0,702 \\
\hline
\end{tabular}

Fuente: Elaboración propia de los autores (2016) 
Se llevó a cabo una regresión múltiple del rendimiento académico a partir de las tres dimensiones de la subescala de hardiness académico y del sexo de los estudiantes. Se obtuvo que el modelo explica significativamente el $14 \%$ de la varianza total de la variable predicha $\left(R^{2}=0,144, F=20,5, p=0,00\right)$. En específico, el sexo predice significativamente al rendimiento académico: las mujeres presentan mayor rendimiento que los hombres $(\beta=0,31, p=0,00)$, aspecto que reafirma resultados anteriores. Las dimensiones de control y compromiso se relacionan significativamente con el rendimiento reportado por los estudiantes ( $\beta=0,13, p=0,003$ y $\beta=0,13, p=0,002$, respectivamente). Así, estudiantes que expresan mayor control y compromiso académico reportan un mayor rendimiento (ver tabla $\mathrm{n} . \stackrel{0}{7}$ ).

Tabla N.O 7

Regresión del rendimiento académico a partir de los componentes del ACP de hardiness académico con los 12 ítems de la prueba de IA

\begin{tabular}{lllll}
\hline & Beta & $\mathrm{t}$ & $\mathrm{p}$ & Regresión \\
\hline (Intercepto) & & 43,295 & 0,00 & $\mathrm{R}=0,38$ \\
Sexo & 0,310 & 7,274 & $0,00^{*}$ & $\mathrm{~F}=20,49$ \\
Asertividad & 0,078 & 1,852 & 0,065 & $\mathrm{P}=0,00^{*}$ \\
Control & 0,125 & 2,973 & $0,003^{*}$ & $\mathrm{~N}=492$ \\
Compromiso & 0,132 & 3,096 & $0,002^{*}$ & \\
& \multicolumn{3}{c}{${ }^{*}$ Significativo al 0,01} \\
\end{tabular}

Fuente: Elaboración propia de los autores (2016)

Por último, se evaluaron las diferencias en las dimensiones de hardiness académico por sexo mediante una prueba t de Student para muestras independientes (ver tabla n. ${ }^{\circ} 8$ ). Hubo diferencias por sexo en las dimensiones asertividad y compromiso, de manera que las mujeres manifestaron mayor asertividad (mujeres $=1,00$ y hombres $=0,98$ ) y los hombres reportaron mayor compromiso (hombres $=1,07$ y mujeres $=0,93$ ). 
Tabla N.O8

ANOVA de una vía de los componentes del ACP

de hardiness académico en función del sexo (puntaje estandarizado de los componentes)

\begin{tabular}{lcccccc}
\hline Componente & \multicolumn{5}{l}{ Homog. $\mathrm{S}^{2}$} & \multicolumn{4}{l}{ Contraste de $\mathrm{H}_{0}$} & $\mathrm{~d}$ \\
\cline { 2 - 6 } & $\mathrm{F}$ & Sig. & $\mathrm{T}$ & $\mathrm{gl}$ & $\mathrm{p}(2$-colas $)$ & \\
Asertividad & 1,01 & 0,315 & 2,84 & 851 & 0,005 & 0,194 \\
Control & 1,59 & 0,208 & 0,874 & 851 & 0,382 & ----- \\
Compromiso & 5,0 & 0,026 & $-4,07$ & 851 & 0,00 & 0,278 \\
& & & & & & \\
\hline
\end{tabular}

Fuente: Elaboración propia de los autores (2016)

\section{DISCUSIÓN}

El objetivo del presente estudio fue elaborar un instrumento de involucramiento académico a partir de los ítems elaborados por Tinio (2009), y realizar un análisis de la confiabilidad, del comportamiento de los ítems, estructura factorial y validez de criterio del instrumento resultante. Se realizó un estudio piloto, con evaluación de jueces expertos, que permitió disminuir los ítems de 102 a 50, al escoger aquellos que se consideraron más relacionados con el IA en los estudiantes y más ajustados al contexto venezolano.

Los análisis de confiabilidad indican que el instrumento es internamente consistente y que todos los ítems se asocian con la media total de IA, lo que permite tener un instrumento con bajo error de medida. A partir del análisis de componentes principales, se obtuvo una estructura compuesta por cinco dimensiones (apego a la universidad, atención en clase, participación activa, dedicación, focalización en la tarea e integración social) que no se corresponde con las estructuras de otros instrumentos, como las reportadas por Appleton, Christenson y Furlong (2008), donde el IA solo parece tener dos componentes (conductual y emocional o afectivo) o tres, si se agrega un elemento cognitivo. Así, la configuración obtenida, más que responder a un aspecto general relacionado con la modalidad de respuesta (afectivo, cognitivo y conductual), responde a las conductas o actividades que realiza el estudiante en el día a día durante su estadía en la universidad, lo que está relacionado de esta manera directamente con el contenido del reactivo.

Autores como Parra (2010) y Kuh et al. (2005) han conseguido estructuras factoriales parecidas, con instrumentos similares de IA. Parra (2010) cuestiona sobre 
conductas relacionadas con vigor, dedicación y absorción, y Kuh et al. (2005) con la cantidad de tiempo y esfuerzo que los estudiantes dedican a sus estudios.

Ahora bien, si se quisiera hacer una correspondencia con la modalidad de respuesta, el primer factor (apego a la universidad) y el sexto (integración social) podrían asociarse con el aspecto afectivo o emocional. Para Tinio (2009), el involucramiento emocional envuelve "las reacciones positivas y negativas hacia las personas y la institución" (p. 65), es decir, los sentimientos y la satisfacción relacionada con la universidad, los profesores y compañeros.

Por otra parte, los factores 2, 3, 4 y 5 (atención en clase, participación activa, dedicación y focalización en la tarea) obtenidos en esta investigación se podrían englobar en el componente conductual. De hecho, si se analizan los reactivos de la agrupación de la presente investigación según la estructura factorial encontrada por Tinio (2009), dichos factores contienen conductas relacionadas con el aprendizaje.

Tinio (2009) propuso tres elementos del factor involucramiento conductual: a) conductas relacionadas con el aprendizaje, b) complacencia con las reglas de la institución escolar y c) participación en actividades extracurriculares. De esta manera, los factores obtenidos en la presente investigación se relacionan con el primer elemento del involucramiento conductual de Tinio (2009): "esfuerzo, concentración, atención, participación y contribución en las discusiones en clases" (p. 65). Estos aspectos permiten afirmar que el instrumento de IA evaluado presenta una estructura factorial consistente, lo que apunta a la validez de su constructo.

Las dimensiones identificadas no logran predecir el rendimiento académico, que fue obtenido por la calificación promedio reportada por los estudiantes, tal como establece la literatura (Astin, 1993; Pace, 1980; Pascarella y Terenzini, 1991; Tejedor, 2003). Se encontró que son trece ítems los que logran predecir significativamente el rendimiento. Tres de ellos pertenecen a la dimensión de apego (me agrada venir a la universidad, me siento seguro en la universidad, aprecio el trabajo arduo que realizan los profesores); uno a la atención (me mantengo concentrado cuando respondo un examen); tres a la participación (doy mi punto de vista acerca del tema en las discusiones en clases, me involucro en actividades extracurriculares, trato de responder a las preguntas del profesor); uno a la dedicación (me esfuerzo por ser un excelente estudiante); tres a la focalización (no me doy por vencido cuando la tarea asignada es difícil, no entrego los trabajos si están incompletos, no me distraigo con mis compañeros durante la clase); y dos a la dimensión integración (me gusta trabajar en grupo, participo en grupos de estudio).

De esta manera, se detectaron elementos específicos de IA que lograron predecir el rendimiento académico reportado, lo que apunta la validez de criterio y permitiría focalizar la atención en estas conductas y sentimientos para incrementar el rendimiento de los estudiantes universitarios. 
Por otro lado, se evidenció que los hombres reportan una mayor participación activa y las mujeres una mayor dedicación a los estudios. Esto concuerda con lo reportado por Finn (1993), quien encontró diferencias por sexo, pero en sentido inverso al hallado en la presente investigación. En el trabajo de Finn (1993), las mujeres presentaron un mayor porcentaje de participación académica y un mayor porcentaje de asistencia que los varones; mientras que los varones presentaron mayor porcentaje de participación extraacadémica. En otro orden, los varones obtuvieron un mayor porcentaje de problemas de conducta, lo cual concuerda con lo encontrado en la presente investigación. Parada y Pérez (2014) también encontraron que el mayor IA lo muestran las estudiantes mujeres.

Contario a los resultados de las investigaciones anteriormente reportadas y de los encontrados en el presente estudio, Newman, Davies y Marder (2003) concluyeron que los estudiantes de sexo masculino tienden a tener menos días de ausentismo que los de sexo femenino; sin embargo, tienden a tener menores niveles de IA. Asimismo, la carrera en cierta forma diferenció al involucramiento. Los estudiantes de comunicación social y derecho presentaron el mayor apego, y los estudiantes de ingeniería y psicología reportaron el menor apego. Por su parte, los estudiantes de derecho y psicología manifestaron poseer mayor atención, mientras que los de ingeniería y administración menor atención en clase. Estos resultados indican la posible influencia de aspectos culturales, institucionales o curriculares asociados a cada carrera. También podría proponerse la hipótesis de que los estudiantes que ingresan a estas carreras son diferentes en los aspectos de involucramiento antes de ingresar a la universidad.

Brint, Cantwell y Hannerman (2008) encontraron diferencias en el IA entre los estudiantes de humanidades y ciencias sociales con los estudiantes de ciencias naturales e ingeniería. Identificaron también que los estudiantes de arte, humanidades y ciencias sociales reportaron más conductas relacionadas con comunicarse con los profesores por correo electrónico, hablar con ellos fuera de clases, contribuir con la discusión en clases, hacer preguntas en conferencias, traer ideas de otros cursos en la discusión en clases y, en ocasiones, encontrar sus cursos muy interesantes y trabajar más de lo necesario. Por el contrario, los estudiantes de ciencias naturales e ingeniería tendían a trabajar en grupos con estudiantes de otras clases para ayudarlos a resolver problemas, manifestaban querer clases que expliquen y enseñen cómo resolver problemas, y buscaban tener un alto nivel de interés en el prestigio y en los empleos bien remunerados. Los autores concluyen que las ciencias sociales y humanísticas presentan una cultura de la afirmación individual, la participación en clases y el interés en las ideas, mientras que la cultura de las ciencias sociales y la ingeniera está basada en el trabajo hacia la competencia cuantitativa mediante el estudio individual y el esfuerzo colaborativo; prevalece el análisis y las habilidades cuantitativas, así como el manejo de computadoras. 
Además, la presente investigación halló que el instrumento permitió establecer, a través del uso de doce reactivos, lo que la literatura denomina como personalidad resistente o hardiness académico (Kobasa, 1979). El análisis de confiabilidad arrojó una adecuada consistencia interna de esta subescala y se encontró una estructura factorial cónsona con la conceptualización establecida por Kobasa et al. (1982) sobre el hardiness académico: compromiso, control y asertividad.

En el presente estudio, estas dimensiones de hardiness académico lograron predecir el rendimiento reportado por los estudiantes. En la literatura sobre el tema, se encuentra que la mayoría de las investigaciones se enfoca en la relación entre ese tipo de personalidad y la salud o aspectos relacionados a esta: estrategias de afrontamiento, burnout, entre otros (Allred y Smith, 1989; Godoy-Izquierdo y Godoy, 2002; Moreno, Garrosa y González, 2000). Algunas investigaciones sobre la relación entre hardiness y rendimiento académico son la de Karimi y Venkatesan (2009) y la de Daneshamooz y Alamolhodaei (2012), quienes encontraron una relación baja positiva, pero significativa, de la fortaleza académica con el rendimiento. Esto indica, al igual que en el presente estudio, que altos puntajes en las dimensiones de personalidad académica resistente se asocian con un alto rendimiento académico. Estos resultados apuntan a considerar el hardiness académico como una variable importante para el estudiante, las instituciones de aprendizaje o de conocimiento, los educadores y las prácticas curriculares.

Las dimensiones de hardiness académico fueron diferenciadas por el sexo de los estudiantes, en el sentido de que las mujeres manifestaron mayor asertividad y los hombres reportaron mayor compromiso. Algunos autores señalan que no existen diferencias en el hardiness académico según el sexo (Daneshamooz y Alamolhodaei, 2012, Karimi y Venkatesan, 2009), pero debe destacarse que en estas investigaciones no se evaluó la fortaleza académica por dimensión, sino como puntaje total, lo que podría explicar las diferencias entre los resultados.

\section{CONCLUSIONES}

Sobre la base de los análisis realizados a la Escala de Involucramiento Académico y la revisión de la literatura, se puede concluir que este instrumento es confiable y válido para la muestra evaluada. Asimismo, la consecución de la subescala de hardiness académico se presenta como un instrumento confiable, válido y relevante para la predicción de rendimiento académico.

El tener un instrumento de este tipo permite, como se indicó, conocer más al estudiantado en cuanto a su IA y facilitar un conjunto de prácticas institucionales que podrían conducir a altos niveles de participación de los estudiantes (Astin, 1993; Chickering y Reisser, 1993; Kuh, et al., 1991; Pascarella y Terenzini, 1991). Estos aspectos coinciden con propuestas como la de los siete principios de buenas prácticas en la educación de pregrado de Chickering y Gamson (1987). Entre estos principios 
destacan: los contactos estudiantes-facultad, la cooperación entre los estudiantes, el aprendizaje activo, la retroalimentación del sistema, el tiempo en la tarea, las altas expectativas y el respeto por diversos talentos y formas de aprendizaje. Son aspectos que se pueden evaluar utilizando el instrumento de involucramiento académico que la presente investigación propone.

El manejo operacional del IA que puede proveer este instrumento proporciona a la universidad otro indicador de la calidad universitaria, distinto de los indicadores tradicionales. A partir de la información recolectada de esta manera, los cuerpos docentes y administrativos podrían organizar el plan de estudios y otros aspectos de la universidad para alentar a los estudiantes a dedicar más esfuerzo en, por ejemplo, escribir más artículos, leer más libros, reunirse con mayor frecuencia con los profesores y compañeros, o utilizar apropiadamente las tecnologías de la información. Todo esto resultará en un mejoramiento en áreas de pensamiento crítico, resolución de problemas, comunicación efectiva y ciudadanía responsable (Kuh, 2001).

Sin embargo, el instrumento presentado y su validación deben evaluarse en otras universidades, dado que la muestra utilizada se limitó a estudiantes de dos universidades privadas. En el caso de que estos estudios adicionales encuentren las mismas dimensiones que las que se reportan en la presente investigación, podrían usarse los reactivos separados de cada una de ellas y evaluarlas según los objetivos específicos de las futuras investigaciones. Se recomienda, además, ampliar la gama de variables a relacionar con el IA, como deserción, prosecución, satisfacción, entre otras.

\section{REFERENCIAS}

Abello, R., Díaz, A., Pérez, M.V., Almeida, L., Lagos, I., González, J., y Strickland, B. (2012). Vivencias e implicación académica en estudiantes universitarios: adaptación y validación de escalas para su evaluación. Estudios Pedagógicos, 38(2), 7-19.

Allred, K., y Smith, T. (1989). The hard personality: Cognitive and pshysiological responses to evaluative threat. Journal of Personality and Social Psychology, 56(2), 257-266.

Appleton, J., Christenson, S., y Furlong, M. (2008). Student engagement with school: critical, conceptual and methodological issues of the construct. Psychology in the Schools, 45(5), 369-386.

Astin, A. W. (1991). Assessment for excellence: The philosophy and practice of assessment and evaluation in higher education. Nueva York, NY: American Council on Education-Macmillan.

Astin, A. W. (1993). What matters in college? Four critical years revisited. San Francisco, CA: Jossey-Bass.

Astin, A. W. (1999). Student involvement: a developmental theory for higher education. Journal of College Student Development, 40(5), 518- 529.

Averill, J. R. (1973). Personal control over aversive stimuli and its relationship to stress. Psychological Bulletin, 80, 286-303.

Brint, S., Cantwell, A., y Hannerman, R. (2008). Two cultures: Undergraduate academic engagement. Research \& Occasional Paper Series, CSHE.4.08. Recuperado de 
http://www.cshe.berkeley.edu/sites/default/files/shared/publications/docs/ROP.Brint.TwoCultures. 03.2.08.pdf

Casuso-Holgado, M.J, Cuesta-Vargas, A.I., Moreno-Morales, N., Labajos-Manzanares, M.T., Barón-López, F.J., y Vega-Cuesta, M. (2013). The association between academic engagement and achievement in health sciences students. BMC Medical Education, 13(33). doi:10.1186/1472-6920-13-33

Chickering, A., y Gamson, Z. (1987). Seven principles for good practice in undergraduate education. $A A H E$ Bulletin, 39(7), 3-7.

Chickering, A., y Reisser, L. (1993). Education and identity (2ª ed.). San Francisco, CA: Jossey-Bass.

Commission on Higher Education. (1996). Framework for outcomes assessment (2 ${ }^{\mathrm{a}}$ ed.). Filadelfia, PA: Middle States Association of Colleges and Schools.

Daneshamooz, S., y Alamolhodaei, H. (2012). Cooperative learning and academia hardiness on students mathematical performance with different levels of mathematics anxiety. Educational Research, 3(3), 207-276.

Dehyadegary, E., Yaacob, S., Juhari, R., y Talib, M. (2011). Academic engagement as a mediator in the relationship between parental school involvement and academic achievement. Australian Journal of Basic and Applied Science, 5(11), 724-729.

Finn, J. (1993). School engagement and students at risk. Washington, DC: National Center for Education Statistics.

Godoy-Izquierdo, D y Godoy, J. (2002). La personalidad resistente: Una revisión de la conceptualización e investigación sobre la dureza. Clínica y Salud, 13(2), 135-162.

Judkins, S. K. (2001). Hardiness, stress and coping strategies among mid-level nurse managers: implications for continuing higher education (Disertación doctoral). Universidad North Texas, Denton, Texas, Estados Unidos.

Karimi, A., y Venkatesan, S. (2009). Mathematics anxiety, mathematics pefomance and academic hardiness in high school students. International Journal of Education and Science, 1(1), 33-37.

Kobasa, S. (1979). Stressful life events, personality and health: an inquiry into hardiness. Journal of Personality and Social Psychology, 37(1), 1-11.

Kobasa, S., Maddi, S. y Kahn S. (1982). Hardiness and health: A prospective study. Journal of Personality and Social Psychology, 42(1), 168 - 177.

Kuh, G. (2001). The national survey of student engagement: conceptual framework and overview of psychometric propeties. Bloomington, IN: Indiana University Center for Postsecondary Research.

Kuh, G. (2009). The national survey of student engagement: conceptual and empirical foundations. New Directions for Institutional Research, 141, 5-20.

Kuh, G., Kinzie, J., Schuh, J., y Whitt, E. (2005). Student success in college. Nueva Jersey, NJ: Wiley, John and Sons. 
Kuh, G., Schuh, J., Whitt, E., y Asociados. (1991). Involving colleges: Successful approaches to fostering student learning and personal development outside the classroom. San Francisco, CA: JosseyBass.

Moreno, B., Garrosa, E., y González, J. (2000). Personalidad resistente, burnout y salud. Escritos de Psicología, 4, 64-77.

Newman, L., Davies, E., y Marder, C. (2003). School engagement of youth with Disabilities. En M. Wagner, C. Marder, J. Blackorby, R. Cameto, L. Newman, P. Levine y E. Davies, The achievements of youth with disabilities during secondary school. A report from the national longitudinal transition study-2 (nlts2). Recuperado de: http://www.nlts2.org/reports/2003 11/nlts2 report 200311 ch3.pdf

Nowack, K. M. (1991). Psychosocial predictors of physical health status. Work and stress, 5, 117-131.

Parada, M. y Pérez, C. (2014). Relación del engagement académico con características académicas y socioafectivas en estudiantes de odontología. Educación Médica Superior, 28(2) 199-215.

Parra, P. (2010). Relación entre el nivel de engagement y el rendimiento académico teórico/práctico. Revista de Educación en Ciencias de la Salud, 7(1), 57-63.

Pascarella, E., y Terenzini, P. (1991). How college affects students: Findings and insights from twenty years of research. San Francisco, CA: Jossey-Bass.

Pace, C.R. (1980). Measuring the quality of student effort. Current Issues in Higher Education, 2, 10-16.

Phares, E. J. (1976). Locus of control in personality. Morristown, NJ: General Learning Press.

Rodríguez, S., Fita, E., y Torrado, M. (2004). El rendimiento académico en la transición secundaria universidad. Revista de Educación, 334, 391-414.

Sbrocco, S. (2009). Student Academic Engagement and the Academic Achievement Gap between Black and White Middle School Students: Does Engagement Increase Student Achievement?

(Disertación doctoral). Recuperado de http://conservancy.umn.edu/bitstream/handle/11299/57326/1/Sbrocco umn 0130E 10755.pdf

Sirin, S.R., y Sirin, L. (2004). Exploring school engagement of middle-class African american adolescents. Youth \& society, 35, 323-340. doi:10.1177/0044118X03255006

Tejedor, F. J. (2003). Poder explicativo de algunos determinantes del rendimiento en los estudios universitarios. Revista Española de Pedagogía, 224, 5-32.

Tinio, M.F. (2009) Academic engagement scale for grade school students. The Assessment Book, 2, 64-75.

Trowler, V., y Trowler, P. (2011). Student Engagement: Conceptual Overview. Londres, Inglaterra: The Leadership Foundation for Higher Education.

Wang, M., y Holcombe, R. (2010). Adolescents' perceptions of school environment, engagement and academic achievement in middle school. American Educational Research Journal, 47, 633- 662. doi:10.3102/0002831209361209

Whitt, E., Asel, A., y Edvalson, S. (2008). Research on lowa student experience: Academic engagement. Recuperado de https://studentsuccess.uiowa.edu/assets/RISE-Brief-Academic-Engagement.pdf 